\title{
Existence and approximation of solutions for generalized extended nonlinear variational inequalities
}

\author{
Balwant Singh Thakur ${ }^{1}$ and Mihai Postolache ${ }^{2^{*}}$
}

\section{"Correspondence:}

emscolar@yahoo.com

${ }^{2}$ Department of Mathematics and

Informatics, University Politehnica of

Bucharest, Bucharest, 060042,

Romania

Full list of author information is

available at the end of the article

\begin{abstract}
In this paper, we consider a new class of generalized extended nonlinear quasi-variational inequality problems involving set-valued relaxed monotone operators and establish its equivalence with the fixed point problem. We study criteria for existence of their solutions. Iterative methods for finding approximate solutions are also proposed and analyzed.

MSC: 47J20; 65K10; 65K15; 90C33

Keywords: generalized extended nonlinear variational inequality; fixed point problem; relaxed monotone operator; relaxed cocoercive mapping; nonexpansive mapping
\end{abstract}

\section{Introduction}

Variational inequality theory constitutes significant and novel extensions of the variational principles. It describes a broad spectrum of interesting developments involving a link between various fields of physical, engineering, pure and applied sciences. It has been shown that variational inequality theory provides the unified and efficient framework for a general treatment of a wide class of problems; for details, see Baiocchi and Capelo [1], Fukushima [2], Giannessi and Maugeri [3], Glowinski and Tallec [4], Noor et al. [5], Patriksson [6], Kinderlehrer and Stampacchia [7] and references therein. The development of variational inequality theory can be viewed as the simultaneous pursuit of two different lines of research. On the one hand, it reveals the fundamental fact on the qualitative aspects of the solutions to important classes of problems; on the other hand, it also enables us to develop highly efficient and powerful new numerical methods for solving various problems. One of the most interesting and important problems in variational inequality theory is the development of efficient numerical methods. There is a substantial number of numerical methods, including the projection methods and their variant forms. The projection method and its variant forms represent important tools for approximate solvability of various kinds of variational inequalities; see [1-34] and references therein. The main idea behind this technique is to establish equivalence between the variational inequalities and the fixed point problem, using the concept of projection. This alternate formulation is used to suggest iterative methods for approximate solvability of variational inequality problems.

O2013 Thakur and Postolache; licensee Springer. This is an Open Access article distributed under the terms of the Creative Commons Attribution License (http://creativecommons.org/licenses/by/2.0), which permits unrestricted use, distribution, and reproduction in any medium, provided the original work is properly cited. 
In many problems of analysis, one encounters operators who may be split in the form $S=A \pm T$, where $A$ and $T$ satisfy some conditions, and $S$ itself has neither of these properties. An early theorem of this type was given by Krasnoselskii [8], where a complicated operator is split into the sum of two simpler operators. There is another setting arising from perturbation theory. Here, the operator equation $T x \pm A x=x$ is considered as a perturbation of $T x=x($ or $A x=x$ ), and one would like to assert that the original unperturbed equation has a solution. In such a situation, there is, in general, no continuous dependence of solutions on the perturbations. For various results in this direction, please see Browder [9], Fucik [10, 11], Kirk [12], Petryshyn [13], Webb [14]. Another argument is concerned with the approximate solution of the problem: For $f$ in $H$, find $x$ in $H$ such that $T x \pm A x=f$. Here $T$ and $A$ are given self-operators of $H$. Many boundary value problems for quasi-linear partial differential equations arising in physics, fluid mechanics and other areas of applications can be formulated as the equation $T x \pm A x=f$; see, e.g., Zeidler [15]. Combettes and Hirstoaga [16] showed that the finding of zeros of sum of two operators can be solved via the variational inequality involving sum of two operators. Several authors have studied this type of situations; see, e.g., Dhage [17], O'Reagan [18] and references therein.

It is our aim in this paper, to consider a new class of generalized extended nonlinear quasi-variational inequality problems, involving set-valued relaxed monotone operators, and to establish its equivalence with the fixed point problem. Using this framework, we study criteria for existence of their solutions. Iterative methods for finding approximate solutions are also proposed and analyzed. As we shall see, in some circumstances, our results reduce to previous results of Bruck [19], Fang and Peterson [20], Lions and Stampacchia [21], Noor [22-24], Verma [25, 26], Qin and Shang [27], Noor and Noor [28, 29].

\section{Preliminaries}

Let $\mathcal{H}$ be a real Hilbert space whose inner product and norm are denoted by $\langle\cdot, \cdot\rangle$ and $\|\cdot\|$, respectively. Let $K: \mathcal{H} \rightarrow \mathcal{H}$ be a point to set mapping, which is closed and convex valued. In other words, for every $x \in \mathcal{H}$, the set $K(x)$ is closed and convex.

We consider the problem of finding $x^{*} \in H$ and $w^{*} \in T\left(x^{*}\right)$ such that $g\left(x^{*}\right) \in K\left(x^{*}\right)$ and

$$
\left\langle\rho\left(A x^{*}+w^{*}\right)+g\left(x^{*}\right)-h\left(x^{*}\right), h\left(y^{*}\right)-g\left(x^{*}\right)\right\rangle \geq 0, \quad \forall h\left(y^{*}\right) \in K\left(x^{*}\right)
$$

for some $\rho>0$, where $A: \mathcal{H} \rightarrow \mathcal{H}$ and $T: \mathcal{H} \rightarrow 2^{\mathcal{H}}$ are nonlinear mappings, while $g, h$ : $\mathcal{H} \rightarrow \mathcal{H}$ are any mappings.

We call inequality (2.1) a generalized extended nonlinear quasi-variational inequality problem.

We now list some special cases of generalized extended nonlinear quasi-variational inequality problem (2.1).

(1) If we take $T=0$, then problem (2.1) is equivalent to the extended general quasi-variational inequality problem introduced and studied by Noor et al. [29, 30].

(2) If we take $T=0$ and $g=I$, then problem (2.1) is equivalent to a class of quasi-variational inequality problems introduced by Noor et al. [29].

(3) If we take $T=0$ and $g=h$, then problem (2.1) is equivalent to the general quasi-variational inequality problem studied by Noor et al. [30]. 
(4) If we take $T=0$ and $h=I$, then problem (2.1) is equivalent to the general quasi-variational inequality problem defined by Noor et al. [28].

If $K\left(x^{*}\right) \equiv K$, that is, the convex set $K\left(x^{*}\right)$ is independent of the solution $x^{*}$, then generalized extended nonlinear quasi-variational inequality problem (2.1) is equivalent to finding $x^{*} \in \mathcal{H}$ and $w^{*} \in T\left(x^{*}\right)$ such that $g\left(x^{*}\right) \in K$ and

$$
\left\langle\rho\left(A x^{*}+w^{*}\right)+g\left(x^{*}\right)-h\left(x^{*}\right), h\left(y^{*}\right)-g\left(x^{*}\right)\right| \geq 0, \quad \forall h\left(y^{*}\right) \in K
$$

for some $\rho>0$, where $K$ is a closed and convex subset of a real Hilbert space $\mathcal{H}$.

We call inequality (2.2) a generalized extended nonlinear variational inequality problem. Variational inequality problem (2.2) covers several variational inequality problems studied in the literature, to which we now turn:

(1) If we take $T=0$, then problem (2.2) is equivalent to the extended general variational inequality problem introduced and studied by Noor [31].

(2) If $T$ is single-valued and $h$ is an identity mapping, then problem (2.2) is equivalent to a variational inequality problem studied by Noor and Noor [28].

(3) If we take $g, h$ as identity mappings, then problem (2.2) reduces to a variational inequality problem studied by Verma [26], Qin et al. [27].

(4) If we take $T=0$ and $g=h$, then problem (2.2) is equivalent to the general variational inequality problem studied by Noor $[23,24]$.

(5) If we take $A=0$ and $h$ as an identity mapping, then problem (2.2) is equivalent to a variational inequality studied by Verma [25].

(6) If $T$ is single-valued and $g, h$ are identity mappings, then problem (2.2) is equivalent to a variational inequality problem studied by Noor [22].

(7) If $A=0$ and $g, h$ are identity mappings, then problem (2.2) is equivalent to a variational inequality problem studied by Bruck [19] and Fang et al. [20].

(8) If $T=0$ and $g, h$ are identity mappings, then problem (2.2) is equivalent to a classical variational inequality problem studied by Lions and Stampacchia [21].

Let us recall the following standard and classical result.

Lemma 2.1 Let $K(x)$ be a closed and convex set in a Hilbert space $\mathcal{H}$. Then, for a given $z \in \mathcal{H}, x \in K(x)$ satisfies the inequality

$$
\langle x-z, y-x\rangle \geq 0, \quad \forall y \in K(x),
$$

if and only if

$$
x=P_{K(x)} z,
$$

where $P_{K(x)}$ is the projection of $\mathcal{H}$ onto the closed convex set $K(x)$ in $\mathcal{H}$.

It is important to point out that the implicit projection operator $P_{K(x)}$ is not nonexpansive. We shall assume that the implicit projection operator $P_{K(x)}$ satisfies the Lipschitz-type continuity, which plays an important and fundamental role in the existence theory and in developing numerical methods for solving the quasi-variational inequalities. 
Assumption 2.1 For all $x, y, z \in \mathcal{H}$, the implicit projection operator $P_{K(x)}$ satisfies the condition

$$
\left\|P_{K(x)} z-P_{K(y)} z\right\| \leq \vartheta\|x-y\|
$$

where $\vartheta$ is a positive constant.

Noor et al. [32] showed that Assumption 2.1 holds for certain cases.

We now recall some definitions.

Definition 2.1 A mapping $A: \mathcal{H} \rightarrow \mathcal{H}$ is said to be:

(i) strongly monotone if there exists a constant $v>0$ such that, for each $x \in \mathcal{H}$,

$$
\langle A(x)-A(y), x-y\rangle \geq v\|x-y\|^{2}
$$

holds for all $y \in \mathcal{H}$;

(ii) $\phi$-cocoercive if there exists a constant $\phi>0$ such that, for each $x \in \mathcal{H}$,

$$
\langle A(x)-A(y), x-y\rangle \geq \phi\|A(x)-A(y)\|^{2}
$$

holds for all $y \in \mathcal{H}$;

(iii) relaxed $\phi$-cocoercive if there exists a constant $\phi>0$ such that, for each $x \in \mathcal{H}$,

$$
\langle A(x)-A(y), x-y\rangle \geq-\phi\|A(x)-A(y)\|^{2}
$$

holds for all $y \in \mathcal{H}$;

(iv) relaxed $(\phi, \gamma)$-cocoercive or relaxed cocoercive with constant $(\phi, \gamma)$ if there exist constants $\phi>0$ and $\gamma>0$ such that, for each $x \in \mathcal{H}$,

$$
\langle A(x)-A(y), x-y\rangle \geq-\phi\|A(x)-A(y)\|^{2}+\gamma\|x-y\|^{2}
$$

holds for all $y \in \mathcal{H}$;

(v) $\mu$-Lipschitz continuous or Lipschitz with constant $\mu$ if there exists a constant $\mu>0$ such that, for each $x, y \in \mathcal{H}$,

$$
\|A(x)-A(y)\| \leq \mu\|x-y\|
$$

(vi) nonexpansive if for each $x, y \in \mathcal{H}$,

$$
\|A(x)-A(y)\| \leq\|x-y\| .
$$

A set-valued mapping $T: \mathcal{H} \rightarrow 2^{\mathcal{H}}$ is said to be:

(vii) $\widehat{H}$-Lipschitz continuous with constant $\zeta$ if there exists a constant $\zeta>0$ such that

$$
\widehat{H}(T(x), T(y)) \leq \zeta\|x-y\|, \quad \forall x, y \in \mathcal{H}
$$


where $\widehat{H}$ is the Hausdorff pseudo-metric, i.e., for any two nonempty subsets $A$ and $B$ of $\mathcal{H}$,

$$
\widehat{H}(A, B)=\max \left\{\sup _{x \in A} d(x, B), \sup _{y \in B} d(A, y)\right\}
$$

where

$$
d(x, B)=\inf _{y \in B}\|x-y\| \quad \text { and } \quad d(A, y)=\inf _{x \in A}\|x-y\| .
$$

It should be pointed out that if the domain of $\widehat{H}$ is restricted to the family of closed bounded subsets of $\mathcal{H}$ (denoted by $C B(\mathcal{H})$ ), then $\widehat{H}$ is the Hausdorff metric.

Lemma 2.2 [35] Let $(X, d)$ be a complete metric space, $T: X \rightarrow C B(X)$ be a set-valued mapping. Then, for any $\varepsilon>0$ and $x, y \in X, u \in T(x)$, there exists $v \in T(y)$ such that

$$
d(u, v) \leq(1+\varepsilon) \widehat{H}(T(x), T(y))
$$

Lemma 2.3 [35] Let $(X, d)$ be a complete metric space, $T: X \rightarrow C B(X)$ be a set-valued mapping satisfying

$$
\widehat{H}(T(x), T(y)) \leq k d(x, y), \quad \forall x, y \in X,
$$

where $0 \leq k<1$ is a constant. Then the mapping $T$ has a fixed point in $X$.

\section{Existence results}

First of all, using Lemma 2.1, we will establish that generalized extended nonlinear quasivariational inequality problem (2.1) is equivalent to a fixed point problem.

Lemma 3.1 $x^{*} \in \mathcal{H}$ and $w^{*} \in T\left(x^{*}\right)$ such that $g\left(x^{*}\right) \in K\left(x^{*}\right)$ is a solution of generalized extended nonlinear quasi-variational inequality problem (2.1) if and only iffor some $\rho>0$, the mapping

$$
F: \mathcal{H} \rightarrow 2^{\mathcal{H}}, \quad F\left(x^{*}\right)=\bigcup_{w^{*} \in T\left(x^{*}\right)}\left\{x^{*}-g\left(x^{*}\right)+P_{K\left(x^{*}\right)}\left(h\left(x^{*}\right)-\rho\left(A\left(x^{*}\right)+w^{*}\right)\right)\right\}
$$

has a fixed point.

Proof Let $x^{*} \in \mathcal{H}$ and $w^{*} \in T\left(x^{*}\right)$ such that $g\left(x^{*}\right) \in K\left(x^{*}\right)$ is a solution of problem (2.1), i.e.,

$$
\left\langle g\left(x^{*}\right)-\left(h\left(x^{*}\right)-\rho\left(A\left(x^{*}\right)+w^{*}\right)\right), h\left(y^{*}\right)-g\left(x^{*}\right)\right| \geq 0
$$

for all $h\left(y^{*}\right) \in K\left(x^{*}\right)$.

Applying Lemma 2.1 to (3.2), we get

$$
g\left(x^{*}\right)=P_{K\left(x^{*}\right)}\left(h\left(x^{*}\right)-\rho\left(A\left(x^{*}\right)+w^{*}\right)\right),
$$


i.e.,

$$
\begin{aligned}
x^{*} & =x^{*}-g\left(x^{*}\right)+P_{K\left(x^{*}\right)}\left(h\left(x^{*}\right)-\rho\left(A\left(x^{*}\right)+w^{*}\right)\right) \\
& \in \bigcup_{w^{*} \in T\left(x^{*}\right)}\left\{x^{*}-g\left(x^{*}\right)+P_{K\left(x^{*}\right)}\left(h\left(x^{*}\right)-\rho\left(A\left(x^{*}\right)+w^{*}\right)\right)\right\} \\
& \Rightarrow x^{*} \in F\left(x^{*}\right),
\end{aligned}
$$

i.e., $x^{*}$ is a fixed point of $F$.

Conversely, let $x^{*}$ be a fixed point of $F$, i.e., $x^{*} \in F\left(x^{*}\right)$, then there exists $w^{*} \in T\left(x^{*}\right)$ such that

$$
x^{*}=x^{*}-g\left(x^{*}\right)+P_{K\left(x^{*}\right)}\left(h\left(x^{*}\right)-\rho\left(A\left(x^{*}\right)+w^{*}\right)\right),
$$

i.e.,

$$
g\left(x^{*}\right)=P_{K\left(x^{*}\right)}\left(h\left(x^{*}\right)-\rho\left(A\left(x^{*}\right)+w^{*}\right)\right) .
$$

Hence,

$$
\left\langle g\left(x^{*}\right)-\left(h\left(x^{*}\right)-\rho\left(A\left(x^{*}\right)+w^{*}\right)\right), h\left(y^{*}\right)-g\left(x^{*}\right)\right| \geq 0 \quad \text { for all } h\left(y^{*}\right) \in K\left(x^{*}\right) .
$$

The proof is complete.

Lemma 3.1 implies that problem (2.1) is equivalent to fixed point problem (3.1). Using this connection, we will establish the following existence result.

Theorem 3.1 Let $A, g, h: \mathcal{H} \rightarrow \mathcal{H}$ be relaxed cocoercive with constants $\left(\phi_{A}, \gamma_{A}\right),\left(\phi_{g}, \gamma_{g}\right)$, $\left(\phi_{h}, \gamma_{h}\right)$ and Lipschitz continuous mappings with constants $\mu_{A}, \mu_{g}, \mu_{h}$, respectively. Let $T$ : $\mathcal{H} \rightarrow C B(\mathcal{H})$ be an $\widehat{H}$-Lipschitz continuous mapping with constant $\zeta>0$. Assume that the following assumption holds:

$$
\begin{aligned}
& \left|\rho-\frac{\gamma_{A}-\phi_{A} \mu_{A}^{2}-\zeta(1-\kappa)}{\mu_{A}^{2}-\zeta^{2}}\right|<\frac{\sqrt{\left(\gamma_{A}-\phi_{A} \mu_{A}^{2}-\zeta(1-\kappa)\right)^{2}-\left(\mu_{A}^{2}-\zeta^{2}\right) \kappa(2-\kappa)}}{\left(\mu_{A}^{2}-\zeta^{2}\right)}, \\
& \mu_{A}^{2}>\zeta^{2}, \quad \gamma_{A}>\zeta(1-\kappa)+\phi_{A} \mu_{A}^{2}+\sqrt{\left(\mu_{A}^{2}-\zeta^{2}\right) \kappa(2-\kappa)}, \\
& 1+\mu_{g}^{2}\left(1+2 \phi_{g}\right)>2 \gamma_{g}, \quad 1+\mu_{h}^{2}\left(1+2 \phi_{h}\right)>2 \gamma_{h},
\end{aligned}
$$

where

$$
\kappa=\vartheta+\sqrt{1-2 \gamma_{g}+\mu_{g}^{2}\left(1+2 \phi_{g}\right)}+\sqrt{1-2 \gamma_{h}+\mu_{h}^{2}\left(1+2 \phi_{h}\right)} .
$$

Then problem (2.1) has a solution.

Proof In the light of Lemma 3.1, it is enough to show that the mapping $F$ defined by (3.1) has a fixed point. For any $x \neq y \in \mathcal{H}, p \in F(x)$ and $q \in F(y)$, there exist $w \in T(x)$ and $u \in T(y)$ 
such that

$$
\begin{aligned}
& p=x-g(x)+P_{K(x)}[h(x)-\rho(A(x)+w)], \\
& q=y-g(y)+P_{K(y)}[h(y)-\rho(A(y)+u)] .
\end{aligned}
$$

Using Assumption 2.1, we have

$$
\begin{aligned}
\|p-q\| \leq & \|x-y-(g(x)-g(y))\| \\
& +\left\|P_{K(x)}[h(x)-\rho(A(x)+w)]-P_{K(y)}[h(y)-\rho(A(y)+u)]\right\| \\
\leq & \|x-y-(g(x)-g(y))\| \\
& \quad+\left\|P_{K(x)}[h(x)-\rho(A(x)+w)]-P_{K(y)}[h(x)-\rho(A(x)+w)]\right\| \\
& \quad+\left\|P_{K(y)}[h(x)-\rho(A(x)+w)]-P_{K(y)}[h(y)-\rho(A(y)+u)]\right\| \\
\leq & \|x-y-(g(x)-g(y))\|+\vartheta\|x-y\|+\|x-y-(h(x)-h(y))\| \\
& +\|x-y-\rho\{A(x)-A(y)\}\|+\rho\|w-u\| .
\end{aligned}
$$

Since $g$ is a relaxed $\left(\phi_{g}, \gamma_{g}\right)$-cocoercive and $\mu_{g}$-Lipschitz continuous mapping, we find the following:

$$
\begin{aligned}
\|x-y-(g(x)-g(y))\|^{2} & =\|x-y\|^{2}-2|g(x)-g(y), x-y\rangle+\|g(x)-g(y)\|^{2} \\
& \leq\left(1+\mu_{g}^{2}\right)\|x-y\|^{2}+2 \phi_{g}\|g(x)-g(y)\|^{2}-2 \gamma_{g}\|x-y\|^{2} \\
& \leq\left(1-2 \gamma_{g}+\mu_{g}^{2}\left(1+2 \phi_{g}\right)\right)\|x-y\|^{2} .
\end{aligned}
$$

Similarly,

$$
\|x-y-(h(x)-h(y))\|^{2} \leq\left(1-2 \gamma_{h}+\mu_{h}^{2}\left(1+2 \phi_{h}\right)\right)\|x-y\|^{2} .
$$

Since $A$ is a relaxed $\left(\phi_{A}, \gamma_{A}\right)$-cocoercive and $\mu_{A}$-Lipschitz continuous mapping, we have

$$
\begin{aligned}
\| x & -y-\rho\{A(x)-A(y)\} \|^{2} \\
& =\|x-y\|^{2}-2 \rho\{A(x)-A(y), x-y\rangle+\rho^{2}\|A(x)-A(y)\|^{2} \\
& \leq\|x-y\|^{2}-2 \rho\left\{-\phi_{A}\|A(x)-A(y)\|^{2}+\gamma_{A}\|x-y\|^{2}\right\}+\rho^{2} \mu_{A}^{2}\|x-y\|^{2} \\
& \leq\|x-y\|^{2}+2 \rho \phi_{A} \mu_{A}^{2}\|x-y\|^{2}-2 \rho \gamma_{A}\|x-y\|^{2}+\rho^{2} \mu_{A}^{2}\|x-y\|^{2} \\
& =\left(1+2 \rho\left(\phi_{A} \mu_{A}^{2}-\gamma_{A}\right)+\rho^{2} \mu_{A}^{2}\right)\|x-y\|^{2} .
\end{aligned}
$$

Now, since $T$ is an $\widehat{H}$-Lipschitz continuous mapping, we estimate

$$
\begin{aligned}
\|w-u\| & \leq(1+\varepsilon) \widehat{H}(T(x)-T(y)) \\
& \leq \zeta(1+\varepsilon)\|x-y\| .
\end{aligned}
$$

Substituting (3.5), (3.6), (3.7) and (3.8) into (3.4), we obtain

$$
\|p-q\| \leq[\kappa+f(\rho)+\rho \zeta(1+\varepsilon)]\|x-y\|
$$


where

$$
\kappa=\vartheta+\sqrt{1-2 \gamma_{g}+\mu_{g}^{2}\left(1+2 \phi_{g}\right)}+\sqrt{1-2 \gamma_{h}+\mu_{h}^{2}\left(1+2 \phi_{h}\right)}
$$

and

$$
f(\rho)=\sqrt{1+2 \rho\left(\phi_{A} \mu_{A}^{2}-\gamma_{A}\right)+\rho^{2} \mu_{A}^{2}} .
$$

By using (3.9), we get

$$
d(p, F(y))=\inf _{q \in F(y)}\|p-q\| \leq(\kappa+f(\rho)+\rho \zeta(1+\varepsilon))\|x-y\|,
$$

since $p \in F(x)$ is arbitrary, we obtain

$$
\sup _{p \in F(x)} d(p, F(y)) \leq(\kappa+f(\rho)+\rho \zeta(1+\varepsilon))\|x-y\| .
$$

Similarly, we get

$$
\sup _{q \in F(y)} d(q, F(x)) \leq(\kappa+f(\rho)+\rho \zeta(1+\varepsilon))\|x-y\|
$$

From the definition of Hausdorff metric $\widehat{H}$, it follows from (3.10) and (3.11) that

$$
\widehat{H}(F(x), F(y)) \leq(\kappa+f(\rho)+\rho \zeta(1+\varepsilon))\|x-y\|, \quad \forall x, y \in \mathcal{H} .
$$

Letting $\varepsilon \rightarrow 0$, we get that

$$
\widehat{H}(F(x), F(y)) \leq(\kappa+f(\rho)+\rho \zeta)\|x-y\|, \quad \forall x, y \in \mathcal{H} .
$$

From (3.3), we get that $(\kappa+f(\rho)+\rho \zeta)<1$, thus $F$ is a set-valued contraction mapping, by Lemma 2.3 it has a fixed point. Lemma 3.1 implies that it is a solution of variational inequality problem (2.1).

\section{Iterative algorithm and convergence}

For given $x_{0} \in \mathcal{H}$ and $w_{0} \in T\left(x_{0}\right)$, let

$$
x_{1}=x_{0}-g\left(x_{0}\right)+P_{K\left(x_{0}\right)}\left(h\left(x_{0}\right)-\rho\left(A\left(x_{0}\right)+w_{0}\right)\right) .
$$

By Lemma 2.3 there exists $w_{1} \in T\left(x_{1}\right)$ such that

$$
\left\|w_{0}-w_{1}\right\| \leq(1+1) \widehat{H}\left(T x_{0}, T x_{1}\right) .
$$

Let $x_{2}=x_{1}-g\left(x_{1}\right)+P_{K\left(x_{1}\right)}\left(h\left(x_{1}\right)-\rho\left(A\left(x_{1}\right)+w_{1}\right)\right)$, then by Lemma 2.3 there exists $w_{2} \in T\left(x_{2}\right)$ such that

$$
\left\|w_{1}-w_{2}\right\| \leq\left(1+\frac{1}{2}\right) \widehat{H}\left(T x_{1}, T x_{2}\right) .
$$

By induction, we can get an iterative algorithm as follows. 
Algorithm 1 For given $x_{0} \in \mathcal{H}, w_{0} \in T\left(x_{0}\right)$, define sequences $\left\{x_{n}\right\}$ and $\left\{w_{n}\right\}$ satisfying

$$
\left.\begin{array}{l}
x_{n+1}=x_{n}-g\left(x_{n}\right)+P_{K\left(x_{n}\right)}\left(h\left(x_{n}\right)-\rho\left(A\left(x_{n}\right)+w_{n}\right)\right), \\
w_{n} \in T\left(x_{n}\right), \quad\left\|w_{n}-w_{n+1}\right\| \leq\left(1+\frac{1}{n+1}\right) \widehat{H}\left(T\left(x_{n}\right), T\left(x_{n+1}\right)\right) .
\end{array}\right\}
$$

Now, we define an Ishikawa-type iterative algorithm [36] for approximate solvability of variational inequality problem (2.2).

Algorithm 2 For a given $x_{0} \in \mathcal{H}$, compute $x_{n+1}$ by the scheme

$$
\begin{aligned}
& y_{n}=\left(1-\beta_{n}\right) x_{n}+\beta_{n}\left[x_{n}-g\left(x_{n}\right)+P_{K\left(x_{n}\right)}\left(h\left(x_{n}\right)-\rho\left(A\left(x_{n}\right)+w_{n}\right)\right)\right], \\
& x_{n+1}=\left(1-\alpha_{n}\right) x_{n}+\alpha_{n}\left[x_{n}-g\left(x_{n}\right)+P_{K\left(y_{n}\right)}\left(h\left(y_{n}\right)-\rho\left(A\left(y_{n}\right)+u_{n}\right)\right)\right],
\end{aligned}
$$

where $w_{n} \in T\left(x_{n}\right), u_{n} \in T\left(y_{n}\right), n=0,1,2, \ldots$ and $\left\{\alpha_{n}\right\},\left\{\beta_{n}\right\}$ are sequences in $[0,1]$, satisfying certain conditions.

To prove the next result, we need the following.

Lemma 4.1 [37] Let $\left\{a_{n}\right\}$ be a nonnegative sequence satisfying

$$
a_{n+1} \leq\left(1-c_{n}\right) a_{n}+b_{n}
$$

with $c_{n} \in[0,1], \sum_{n=0}^{\infty} c_{n}=\infty, b_{n}=o\left(c_{n}\right)$. Then $\lim _{n \rightarrow \infty} a_{n}=0$.

Theorem 4.1 Let $A, T, g$, h satisfy all the assumptions of Theorem 3.1, and let $\left\{\alpha_{n}\right\},\left\{\beta_{n}\right\}$ be sequences in $[0,1]$, for all $n \geq 0$, such that $\sum_{n=0}^{\infty} \alpha_{n}=\infty$. Then the approximate sequences $\left\{x_{n}\right\},\left\{w_{n}\right\}$ constructed by Algorithm 2 converge strongly to a solution of problem (2.1).

Proof By Theorem 3.1, generalized extended nonlinear quasi-variational inequality problem (2.1) has a solution. Let $x^{*} \in \mathcal{H}, w^{*} \in T\left(x^{*}\right)$ such that $g\left(x^{*}\right) \in K\left(x^{*}\right)$ be a solution of (2.1). By Lemma 3.1, we have

$$
x^{*}=x^{*}-g\left(x^{*}\right)+P_{K\left(x^{*}\right)}\left(h\left(x^{*}\right)-\rho\left(A\left(x^{*}\right)-w^{*}\right)\right) .
$$

Using (4.2), we have

$$
\begin{aligned}
\left\|x_{n+1}-x^{*}\right\|= & \left\|\left(1-\alpha_{n}\right) x_{n}+\alpha_{n}\left[x_{n}-g\left(x_{n}\right)+P_{K\left(y_{n}\right)}\left(h\left(y_{n}\right)-\rho\left(A\left(y_{n}\right)+u_{n}\right)\right)\right]-x^{*}\right\| \\
\leq & \left(1-\alpha_{n}\right)\left\|x_{n}-x^{*}\right\| \\
& +\alpha_{n}\left\|\left[x_{n}-g\left(x_{n}\right)+P_{K\left(y_{n}\right)}\left(h\left(y_{n}\right)-\rho\left(A\left(y_{n}\right)+u_{n}\right)\right)\right]-x^{*}\right\| \\
\leq & \left(1-\alpha_{n}\right)\left\|x_{n}-x^{*}\right\|+\alpha_{n}\left\|x_{n}-x^{*}-\left(g\left(x_{n}\right)-g\left(x^{*}\right)\right)\right\| \\
& +\alpha_{n}\left\|P_{K\left(y_{n}\right)}\left[h\left(y_{n}\right)-\rho\left(A\left(y_{n}\right)+u_{n}\right)\right]-P_{K\left(x^{*}\right)}\left[h\left(x^{*}\right)-\rho\left(A\left(x^{*}\right)+w^{*}\right)\right]\right\| \\
\leq & \left(1-\alpha_{n}\right)\left\|x_{n}-x^{*}\right\|+\alpha_{n}\left\|x_{n}-x^{*}-\left(g\left(x_{n}\right)-g\left(x^{*}\right)\right)\right\| \\
& +\alpha_{n}\left\|P_{K\left(y_{n}\right)}\left[h\left(y_{n}\right)-\rho\left(A\left(y_{n}\right)+u_{n}\right)\right]-P_{K\left(y_{n}\right)}\left[h\left(x^{*}\right)-\rho\left(A\left(x^{*}\right)+w^{*}\right)\right]\right\|
\end{aligned}
$$




$$
\begin{aligned}
& +\alpha_{n}\left\|P_{K\left(y_{n}\right)}\left[h\left(x^{*}\right)-\rho\left(A\left(x^{*}\right)+w^{*}\right)\right]-P_{K\left(x^{*}\right)}\left[h\left(x^{*}\right)-\rho\left(A\left(x^{*}\right)+w^{*}\right)\right]\right\| \\
\leq & \left(1-\alpha_{n}\right)\left\|x_{n}-x^{*}\right\|+\alpha_{n}\left\|x_{n}-x^{*}-\left(g\left(x_{n}\right)-g\left(x^{*}\right)\right)\right\| \\
& +\alpha_{n}\left\|h\left(y_{n}\right)-h\left(x^{*}\right)-\rho\left\{\left(A\left(y_{n}\right)+u_{n}\right)-\left(A\left(x^{*}\right)+w^{*}\right)\right\}\right\| \\
& +\alpha_{n} \vartheta\left\|y_{n}-x^{*}\right\| \\
\leq & \left(1-\alpha_{n}\right)\left\|x_{n}-x^{*}\right\|+\alpha_{n}\left\|x_{n}-x^{*}-\left(g\left(x_{n}\right)-g\left(x^{*}\right)\right)\right\| \\
& +\alpha_{n}\left\|y_{n}-x^{*}-\left(h\left(y_{n}\right)-h\left(x^{*}\right)\right)\right\| \\
& +\alpha_{n}\left\|y_{n}-x^{*}-\rho\left(A\left(y_{n}\right)-A\left(x^{*}\right)\right)\right\| \\
& +\alpha_{n} \rho\left\|u_{n}-w^{*}\right\|+\alpha_{n} \vartheta\left\|y_{n}-x^{*}\right\| \\
\leq & \left(1-\alpha_{n}\right)\left\|x_{n}-x^{*}\right\|+\alpha_{n} \sqrt{1-2 \gamma_{g}+\mu_{g}^{2}\left(1+2 \phi_{g}\right)}\left\|x_{n}-x^{*}\right\| \\
& +\alpha_{n} \sqrt{1-2 \gamma_{h}+\mu_{h}^{2}\left(1+2 \phi_{h}\right)\left\|y_{n}-x^{*}\right\|} \\
& +\alpha_{n} \sqrt{1+2 \rho\left(\phi_{A} \mu_{A}^{2}-\gamma_{A}\right)+\rho^{2} \mu_{A}^{2}}\left\|y_{n}-x^{*}\right\| \\
& +\alpha_{n} \rho \zeta(1+\varepsilon)\left\|y_{n}-x^{*}\right\|+\alpha_{n} \vartheta\left\|y_{n}-x^{*}\right\| \\
= & \left(1-\alpha_{n}\right)\left\|x_{n}-x^{*}\right\|+\alpha_{n} \theta_{g}\left\|x_{n}-x^{*}\right\| \\
& +\alpha_{n}\left(\theta_{h}+f(\rho)+\rho \zeta(1+\varepsilon)+\vartheta\right)\left\|y_{n}-x^{*}\right\|,
\end{aligned}
$$

where

$$
\theta_{g}=\sqrt{1-2 \gamma_{g}+\mu_{g}^{2}\left(1+2 \phi_{g}\right)}, \quad \theta_{h}=\sqrt{1-2 \gamma_{h}+\mu_{h}^{2}\left(1+2 \phi_{h}\right)}
$$

and

$$
f(\rho)=\sqrt{1+2 \rho\left(\phi_{A} \mu_{A}^{2}-\gamma_{A}\right)+\rho^{2} \mu_{A}^{2}} .
$$

Similarly, we have

$$
\begin{aligned}
\left\|y_{n}-x^{*}\right\| \leq & \left(1-\beta_{n}\right)\left\|x_{n}-x^{*}\right\|+\beta_{n}\left\|x_{n}-x^{*}-\left(g\left(x_{n}\right)-g\left(x^{*}\right)\right)\right\| \\
& +\beta_{n}\left\|P_{K\left(x_{n}\right)}\left[h\left(x_{n}\right)-\rho\left(A\left(x_{n}\right)+w_{n}\right)\right]-P_{K\left(x^{*}\right)}\left[h\left(x^{*}\right)-\rho\left(A\left(x^{*}\right)-w^{*}\right)\right]\right\| \\
\leq & \left(1-\beta_{n}\right)\left\|x_{n}-x^{*}\right\|+\beta_{n} \theta_{g}\left\|x_{n}-x^{*}\right\| \\
& +\beta_{n}\left\|P_{K\left(x_{n}\right)}\left[h\left(x_{n}\right)-\rho\left(A\left(x_{n}\right)+w_{n}\right)\right]-P_{K\left(x_{n}\right)}\left[h\left(x^{*}\right)-\rho\left(A\left(x^{*}\right)-w^{*}\right)\right]\right\| \\
& +\beta_{n}\left\|P_{K\left(x_{n}\right)}\left[h\left(x^{*}\right)-\rho\left(A\left(x^{*}\right)-w^{*}\right)\right]-P_{K\left(x^{*}\right)}\left[h\left(x^{*}\right)-\rho\left(A\left(x^{*}\right)-w^{*}\right)\right]\right\| \\
\leq & \left(1-\beta_{n}\right)\left\|x_{n}-x^{*}\right\|+\beta_{n} \theta_{g}\left\|x_{n}-x^{*}\right\| \\
& +\beta_{n}\left\|h\left(x_{n}\right)-h\left(x^{*}\right)-\rho\left\{\left(A\left(x_{n}\right)+w_{n}\right)-\left(A\left(x^{*}\right)-w^{*}\right)\right\}\right\| \\
& +\beta_{n} \vartheta\left\|x_{n}-x^{*}\right\| \\
\leq & \left(1-\beta_{n}\right)\left\|x_{n}-x^{*}\right\|+\beta_{n} \theta_{g}\left\|x_{n}-x^{*}\right\|+\beta_{n} \vartheta\left\|x_{n}-x^{*}\right\| \\
& +\beta_{n}\left\|x_{n}-x^{*}-\left(h\left(x_{n}\right)-h\left(x^{*}\right)\right)\right\| \\
& +\beta_{n}\left\|x_{n}-x^{*}-\rho\left\{A\left(x_{n}\right)-A\left(x^{*}\right)\right\}\right\|+\beta_{n} \rho\left\|w_{n}-w^{*}\right\|
\end{aligned}
$$




$$
\begin{aligned}
\leq & \left(1-\beta_{n}\right)\left\|x_{n}-x^{*}\right\|+\beta_{n} \theta_{g}\left\|x_{n}-x^{*}\right\| \\
& +\beta_{n}\left(\theta_{h}+f(\rho)+\rho \zeta(1+\varepsilon)+\vartheta\right)\left\|x_{n}-x^{*}\right\| \\
= & \left(1-\beta_{n}\right)\left\|x_{n}-x^{*}\right\|+\beta_{n}(\kappa+f(\rho)+\rho \zeta(1+\varepsilon))\left\|x_{n}-x^{*}\right\| .
\end{aligned}
$$

Substituting (4.4) into (4.3) yields that

$$
\begin{aligned}
\left\|x_{n+1}-x^{*}\right\| \leq & \left(1-\alpha_{n}\right)\left\|x_{n}-x^{*}\right\|+\alpha_{n} \theta_{g}\left\|x_{n}-x^{*}\right\| \\
& +\alpha_{n}\left(\theta_{h}+f(\rho)+\rho \zeta(1+\varepsilon)+\vartheta\right) \\
& \times\left\{1-\beta_{n}(1-(\kappa+f(\rho)+\rho \zeta(1+\varepsilon)))\right\}\left\|x_{n}-x^{*}\right\| .
\end{aligned}
$$

Letting $\varepsilon \rightarrow 0$, we get from (4.5) that

$$
\begin{aligned}
\left\|x_{n+1}-x^{*}\right\| \leq & \left(1-\alpha_{n}\right)\left\|x_{n}-x^{*}\right\|+\alpha_{n} \theta_{g}\left\|x_{n}-x^{*}\right\| \\
& +\alpha_{n}\left(\theta_{h}+f(\rho)+\rho \zeta+\vartheta\right)\left\{1-\beta_{n}(1-(\kappa+f(\rho)+\rho \zeta))\right\}\left\|x_{n}-x^{*}\right\| \\
\leq & \left(1-\alpha_{n}\right)\left\|x_{n}-x^{*}\right\|+\alpha_{n}\left(\theta_{g}+\theta_{h}+f(\rho)+\rho \zeta+\vartheta\right)\left\|x_{n}-x^{*}\right\| \\
= & \left(1-\alpha_{n}\right)\left\|x_{n}-x^{*}\right\|+\alpha_{n}(\kappa+f(\rho)+\rho \zeta)\left\|x_{n}-x^{*}\right\| \\
= & {\left[1-\alpha_{n}\{1-(\kappa+f(\rho)+\rho \zeta)\}\right]\left\|x_{n}-x^{*}\right\| . }
\end{aligned}
$$

By virtue of Lemma 4.1, we get from (4.6) that $\lim _{n \rightarrow \infty}\left\|x_{n+1}-x^{*}\right\|=0$, i.e., $x_{n} \rightarrow x^{*}$, as $n \rightarrow \infty$. Since

$$
\left\|w_{n}-w^{*}\right\| \leq(1+\varepsilon) \zeta\left\|x_{n}-x^{*}\right\|
$$

letting $n \rightarrow \infty$, we get that $w_{n} \rightarrow w^{*}$. This completes the proof.

Remark 1 For a suitable and appropriate choice of the operators $T, A, g, h$ and $\left\{\alpha_{n}\right\},\left\{\beta_{n}\right\}$, $\left\{\gamma_{n}\right\}$, one can obtain a number of new and previously known iterative schemes for approximate solvability of variational inequality problems as discussed in special cases. This clearly shows that Algorithm 2 is quite general and unifies several algorithms.

Remark 2 Results presented in the paper are significant improvement and extension of the results obtained previously by many authors. Especially, our Theorem 3.1 extends the existence of solution in the literature to the case of generalized extended nonlinear variational inequality (2.1). Algorithm 2 is a very general and unified algorithm for finding an approximate solution of problem (2.1).

\section{Conclusion}

In this paper, we have considered a new class of generalized extended nonlinear quasivariational inequalities, which involves sum of two operators $A: \mathcal{H} \rightarrow \mathcal{H}$ and $T: \mathcal{H} \rightarrow 2^{\mathcal{H}}$. We have established the equivalence between the generalized extended nonlinear variational inequality and the fixed point problem using projection mapping. Using this equivalence, we have first established criteria for the existence of solution of the proposed variational inequality problem. We have also suggested and analyzed some iterative methods 
for approximate solvability of generalized extended nonlinear quasi-variational inequalities. Several special cases of the proposed variational inequality problem have also been discussed.

\section{Competing interests}

The authors declare that they have no competing interests.

\section{Authors' contributions}

All authors contributed equally and significantly in writing this paper. All authors read and approved the final manuscript.

\section{Author details}

'School of Studies in Mathematics, Pt. Ravishankar Shukla University, Raipur, 492010, India. ${ }^{2}$ Department of Mathematics and Informatics, University Politehnica of Bucharest, Bucharest, 060042, Romania.

\section{Acknowledgements}

We are greatly indebted to the anonymous referee for helpful comments and stimulating hints. The first author is supported by the University Grants Commission of India project F. No. 41-1390/2012.

Received: 28 August 2013 Accepted: 20 November 2013 Published: 17 Dec 2013

\section{References}

1. Baiocchi, C, Capelo, A: Variational and Quasi Variational Inequalities. Wiley, New York (1984)

2. Fukushima, M: The primal Douglas-Rachford splitting algorithm for a class of monotone mappings with application to the traffic equilibrium problem. Math. Program., Ser. A 72, 1-15 (1996)

3. Giannessi, F, Maugeri, A (eds.): Variational Inequalities and Network Equilibrium Problems. Plenum, New York (1995)

4. Glowinski, R, Le Tallec, P: Augmented Lagrangian and Operator-Splitting Methods in Nonlinear Mechanics. SIAM Studies in Applied Mathematics, vol. 9. SIAM, Philadelphia (1989)

5. Noor, MA, Noor, Kl, Rassias, TM: Some aspects of variational inequalities. J. Comput. Appl. Math. 47, 285-312 (1993)

6. Patriksson, M: Nonlinear Programming and Variational Inequality Problems. A Unified Approach. Applied Optimization, vol. 23. Kluwer Academic, Dordrecht (1999)

7. Kinderlehrer, D, Stampacchia, G: Introduction to Variational Inequalities and Their Applications. Academic Press, New York (1980)

8. Krasnoselskii, MA: Two remarks of the method of successive approximations. Usp. Mat. Nauk 10, $123-127$ (1955)

9. Browder, FE: Fixed point theorems for nonlinear semicontractive mappings in Banach spaces. Arch. Ration. Mech. Anal. 21, 259-269 (1966)

10. Fucik, S: Fixed point theorems for a sum of nonlinear mapping. Comment. Math. Univ. Carol. 9, 133-143 (1968)

11. Fucik, S: Solving of nonlinear operator equations in Banach space. Comment. Math. Univ. Carol. 10, 177-186 (1969)

12. Kirk, WA: On nonlinear mappings of strongly semicontractive type. J. Math. Anal. Appl. 27, 409-412 (1969)

13. Petryshyn, WV: Remarks on fixed point theorems and their extensions. Trans. Am. Math. Soc. 126, 43-53 (1967)

14. Webb, JRL: Fixed point theorems for nonlinear semicontractive operators in Banach spaces. J. Lond. Math. Soc. 1, 683-688 (1969)

15. Zeidler, V: Nonlinear Functional Analysis and Its Applications, II/B: Nonlinear Monotone Operators. Springer, New York (1990)

16. Combettes, PL, Hirstoaga, SA: Visco-penalization of the sum of two monotone operators. Nonlinear Anal. 69, 579-591 (2008)

17. Dhage, BC: Remarks on two fixed-point theorems involving the sum and the product of two operators. Comput. Math. Appl. 46, 1779-1785 (2003)

18. O'Regan, D: Fixed point theory for the sum of two operators. Appl. Math. Lett. 9, 1-8 (1996)

19. Bruck, RE: An iterative solution of a variational inequality for certain monotone operator in a Hilbert space. Bull. Am. Math. Soc. 81, 890-892 (1975). Corrigendum in vol. 82, p.353, 1976

20. Fang, SC, Peterson, EL: Generalized variational inequalities. J. Optim. Theory Appl. 38, 363-383 (1982)

21. Lions, J, Stampacchia, G: Variational inequalities. Commun. Pure Appl. Math. 20, 493-519 (1967)

22. Noor, MA: On variational inequalities. PhD thesis, Brunel University, London (1975)

23. Noor, MA: General variational inequalities. Appl. Math. Lett. 1, 119-121 (1988)

24. Noor, MA: New extragradient-type methods for general variational inequalities. J. Math. Anal. Appl. 277, 379-394 (2003)

25. Verma, RU: Iterative algorithms for variational inequalities and associated nonlinear equations involving relaxed Lipschitz operators. Appl. Math. Lett. 9,61-63 (1996)

26. Verma, RU: Generalized variational inequalities involving multivalued relaxed monotone operators. Appl. Math. Lett. 10, 107-109 (1997)

27. Qin, $X L$, Shang, M: Generalized variational inequalities involving relaxed monotone mappings and nonexpansive mappings. J. Inequal. Appl. (2007). doi:10.1155/2007/20457

28. Noor, MA, Noor, Kl: On general quasi-variational inequalities. J. King Saud Univ., Sci. 24, 81-88 (2012)

29. Noor, MA, Noor, Kl: Sensitivity analysis of some quasi variational inequalities. J. Adv. Math. Stud. 6(1), 43-52 (2013)

30. Noor, MA, Noor, KI, Khan, AG: Some iterative schemes for solving extended general quasi variational inequalities. Appl. Math. Inform. Sci. 7, 917-925 (2013)

31. Noor, MA: Extended general variational inequalities. Appl. Math. Lett. 22, 182-186 (2009)

32. Noor, MA: On general quasi-variational inequalities. J. King Saud Univ., Sci. 24, 81-88 (2012)

33. Noor, MA: Projection iterative methods for extended general variational inequalities. J. Appl. Math. Comput. 32, 83-95 (2010) 
34. Verma, RU: Generalized system for relaxed cocoercive variational inequalities and projection methods. J. Optim. Theory Appl. 121(1), 203-210 (2004)

35. Nadler, SB: Multi-valued contraction mappings. Pac. J. Math. 30, 475-488 (1969)

36. Ishikawa, S: Fixed points by a new iteration method. Proc. Am. Math. Soc. 44, 147-150 (1974)

37. Weng, XL: Fixed point iteration for local strictly pseudo-contractive mappings. Proc. Am. Math. Soc. 113, 727-731 (1991)

10.1186/1029-242X-2013-590

Cite this article as: Thakur and Postolache: Existence and approximation of solutions for generalized extended nonlinear variational inequalities. Journal of Inequalities and Applications 2013, 2013:590

Submit your manuscript to a SpringerOpen ${ }^{\odot}$ journal and benefit from:

- Convenient online submission

Rigorous peer review

- Immediate publication on acceptance

Open access: articles freely available online

- High visibility within the field

- Retaining the copyright to your article 